\title{
Communication and Communication Problems Between Autonomous Vehicles and Human Drivers
}

\author{
Berthold Färber
}

\subsection{Introduction}

Discussions of autonomous land vehicles often invoke the example of air traffic, where the autopilot is responsible for steering except for take-off and landing. The question arises: what can we learn from air traffic? What autonomously flying aircraft and autonomously driving vehicles have in common is that the pilot or driver bears the final responsibility. But, there are a number of differences between road traffic and air traffic (besides their type of locomotion) that make transferring the systems from one to the other impractical. Two key differences that are central to this article are the application of rules and the form of supervision. Encounters between airplanes - primarily while taxiing - are thoroughly governed by strict rules. In addition, there is a supervisory monitoring and guiding authority that gives pilots precise instructions and, in cases that might not be covered by rules, reaches decisions and communicates with pilots. As such, the pilot does not have any room for discretion or independent decision-making. The pilot's role is purely implementation. Unlike air traffic, road traffic is a self-organizing, chaotic system that, although it is fundamentally governed by rules, includes many situations for which unambiguous rules cannot be determined. In those situations, section 1 of the German Highway Code always applies: "Participating in road traffic requires constant caution and mutual consideration," and "Those who participate in traffic shall behave such that no one else is harmed, endangered, or even disabled or harassed more than inevitable in the circumstances." Thus, even a list of rules for behavior and all conceivable situations as exhaustive as the Highway Code has a large "miscellaneous" category that must be

B. Färber $(\square)$

Bundeswehr Universität München, 85577 Neubiberg, Germany

e-mail: berthold.faerber@unibw.de 
resolved by road users themselves in accordance with section 1 of the Highway Code. It follows that one of a driver's central responsibilities in order to safely participate in road traffic is to assess other road users' behavior. This assessment and prediction of other road users' behavior relies on the initial assumption of more or less rule-abiding behavior on their part. However, another aspect of evaluating intentions involves communication between drivers and road users via actions and gestures. In other words, in addition to the "official" rules, there exists a set of informal rules that help to guide traffic.

Anyone who has visited another country as a tourist, whether as a driver or a pedestrian, has experienced for themselves how drastically informal rules, culturally specific modes of behavior, and communication between road users affect traffic. Human drivers who encounter such divergent "canons of rules" initially react with a phase of irritation. In the second phase, the adaptation phase, they unconsciously adapt over time to these new imprecise and unwritten rules. In the consolidation phase these rules seem to be obvious although they are not set in stone and in some cases cannot even be precisely described.

Motorized road traffic comprises a system with very diverse players whose goal is to convey road users to their destinations safely, quickly, and without incident. From the systemic perspective, it is immediately evident that observance of informal rules, communication between road users, and predictions of others' behavior fulfill two key functions: they facilitate the flow of traffic and help to compensate for errors.

\subsection{Questions}

Expressed technically, humans are multi-sensory adaptive systems. That means that they are capable of taking signals that might be weak or ambiguous and extrapolating the big picture, which they can then interpret. They are also able to adapt to changing conditions in order to support the two aforementioned functions, compensating for one another's errors and improving the flow of traffic. Yet what happens when, alongside humans, there are also robotic vehicles participating in traffic that abide strictly by rules but do not understand informal rules or aspects of communication? How would human drivers react to these new road users, especially in the transition phase when robotic vehicles remain a minority? Are humans capable of solving difficult or seemingly unresolvable situations in cooperation with robotic vehicles? Finally, which characteristics or markings do robotic vehicles need to have at the minimum in order to participate in mixed traffic with human drivers without causing problems?

This article will approach these questions from various angles. Since autonomous vehicles have so far primarily been investigated in view of technical performance, as in the Urban Challenge [1] or the Bertha Benz drive [2], and an emergency driver has always been present in mixed traffic to intervene if necessary (e.g. at the Stadtpilot [3]), there is scarcely any original literature on the questions described above. For that reason, much will be extrapolated from the findings of other research fields. 


\subsection{How do Road Users Communicate?}

In addition to the standard signals for expressing an intention, such as turn signals, emergency lights, brake lights, the horn, and headlight flashing, road users communicate via a number of "informal" communication channels. This kind of communication in road traffic is characterized by restricted comprehensibility compared to normal human communication. According to Merten [4], there are various communication options.

\section{Schema Formation}

In schema formation, a road user's behavior is anticipated based on specific characteristics. For example, an elderly person with mobility issues behaves differently from a child. One expects different driving behavior from the driver of a sports car to that of the driver of a large sedan. Of course, these schemata are not always accurate in day-to-day driving. Nevertheless, they do serve as guiding principles and help to stabilize the entire system of traffic.

\section{Anticipatory Behavior}

Small actions make the direction of someone's behavior predictable for other road users. For example, if a vehicle approaches the left lane (without engaging the turn signal), this indicates an intention to change lanes. Similarly, if a pedestrian purposefully approaches a crosswalk, the driver will assume that the pedestrian would like to cross the street.

\section{Non-verbal Communication}

Non-verbal communication plays a role among the informal communication channels, especially in "negotiation situations." Non-verbal communication is indubitably the oldest form of communication between living beings. As far back as 1874, Charles Darwin discussed non-verbal communication in his book The Expression of the Emotions in Man and Animals [5]. The subtlety of non-verbal signals was demonstrated by an old investigation by Pfungst [6] of Clever Hans, a horse that was able to perform "arithmetic" based on unconscious, minimal signals from its owner or the audience. The important finding was that non-verbal signals are even sent out unconsciously and are thus not always easily available for analysis.

In general, non-verbal signals can be divided into three types:

- Facial expression and eye contact

- Gestures and body movements

- Voice and tone of speech.

In the case of road traffic, only facial expression/eye contact and gestures/body movements are relevant and will therefore be addressed here. 


\section{Facial expressions and eye contact}

In local traffic, eye contact both between drivers and between drivers and other road users (pedestrians and cyclists) plays a critical role. Pedestrians who want to cross the street without a crossing aid use eye contact to ensure that an approaching driver sees them. If the driver returns the eye contact, pedestrians assume that they have been seen and that the driver will act accordingly [7].

When a driver from a side street wants to merge onto a main street with heavier traffic, the merging driver also uses eye contact to make sure he or she can turn despite the short time gap, as the driver on the busier street will reduce speed accordingly.

Eye contact is a two-way form of communication. In other words, a glance is either reciprocated or not reciprocated by the person who is glanced at. If that person looks away, this sends the message that he or she has "not seen" the other person and does not intend to accept the signal or the negotiation. The implications of the strategy for autonomous vehicles should be distinguished between autonomous vehicles in which the driver's seat is occupied and those where it is not.

If the driver's seat is occupied, there are two possibilities:

1. The "driver," i.e. the person in the driver's seat, is preoccupied with other activities inside the vehicle and eye contact does not occur. In that case, the other road user cannot assume that their negotiation proposal has been accepted and will act accordingly.

2. The "driver" of the autonomous vehicle is looking outside and makes incidental eye contact with the other road user. In that case the eye contact with non-autonomous road user can lead to an inaccurate understanding of the situation and thus cause a conflict.

If the driver's seat is not occupied, the other road user does not receive any information, so the situation is equivalent to the "no eye contact" scenario.

\section{Gestures and body movements}

Gestures are a pervasive and effective method of communication between road users. Many signals conveyed using gestures are generally comprehensible and mostly clear. For instance, nodding signifies agreement with the other person's request. Pedestrians move their arms up and down to request vehicles to stop, for instance in order to secure the scene of an accident. Moving one's hands downwards (Fig. 7.1) signifies that the other person should slow down. A sweeping hand movement (Fig. 7.2) or a gesture of offering (Fig. 7.3) with the palm facing upward signifies "Go ahead. I cede my right-of-way." 
Fig. 7.1 Slow down. Image rights: Risser [8]

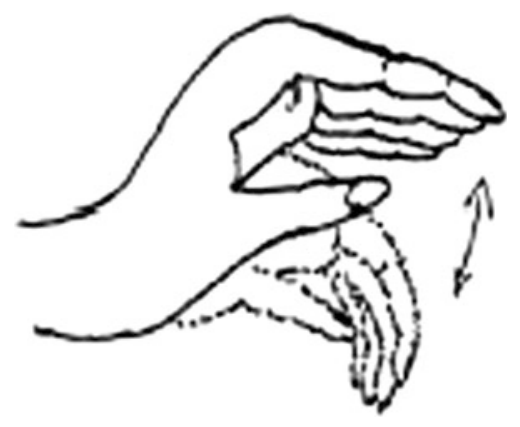

Fig. 7.2 Sweeping hand movement. Image rights: Risser [8]

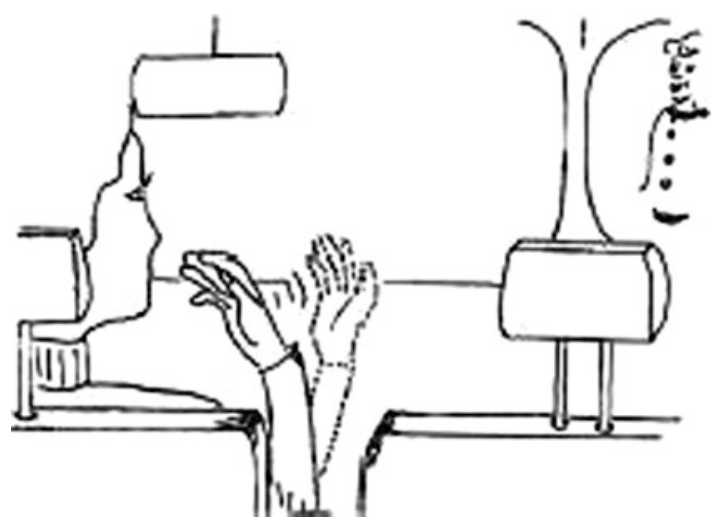

Fig. 7.3 Gesture of offering

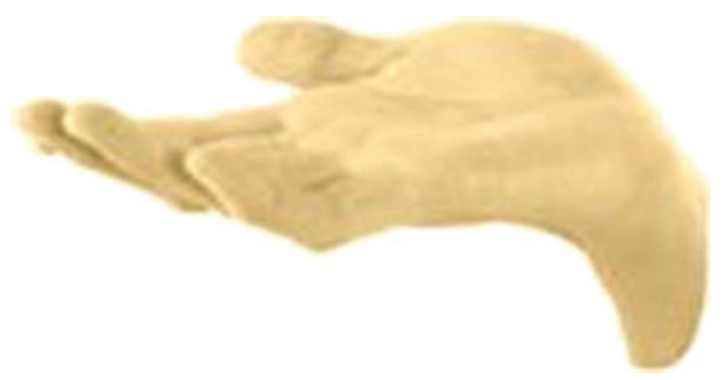

\subsection{Implications of Communications Options on Traffic Safety}

At first glance, informal signals such as eye contact, gestures, or anticipatory behavior seem less clear-cut than standardized signals such as turn signals, the horn, headlight flashing, etc. In fact neither is entirely clear-cut. Interestingly, the philosopher Heidegger, who investigates the nature of symbols in his treatise Being and Time [9], chooses cars as an example: "Motor cars are equipped with an adjustable red arrow whose position 
indicates which direction the car will take, for example, at an intersection" (cit. after Frerichs, [10], p. 138. Perhaps this symbol was still crystal-clear and situationindependent in those early days of cars, but it quickly becomes apparent that symbols take on different meanings in different contexts. All symbols, both verbal and non-verbal, can only be understood in relation to their context. As Savigny [11] explains, a distinction must be made between the signal and the intended meaning. Headlight flashing may have different intended meanings depending on the situation. If a driver who has right-of-way slows down and flashes her headlights, it is interpreted as an offer to yield right-of-way; if she is accelerating or maintaining speed when she makes the same signal, it means that she is asserting her right-of-way. Likewise, a right-turn signal might mean "I'm turning" or "I'm looking for a parking space-go ahead and pass me."

To consider the vulnerable road users, let us take a look at the interesting example of cyclists. Cyclists still use signals from the early days of motorized driving to indicate their intended change in direction. This means that for pattern recognition outside of highway traffic, an automated system needs the ability to identify both cyclists' presence and the signals they make.

What are the implications for traffic safety? Initially we may assume that autonomous vehicles will not be familiar with the assorted context-dependent meanings of signals and must therefore behave very cautiously. As long as visual recognition or a pedestrian's hand signals cannot be reliably perceived and interpreted based on the situation, any pedestrians located on a potential collision course with the vehicle must be treated as hazards and trigger a suitable reaction by the autonomous vehicle.

So when autonomous vehicles identify pedestrians on the roadway, they will stop. What happens if the pedestrian is a police officer directing traffic? For one thing, the police officer must be identified as an upright obstacle that the robotic vehicle must steer around. Beyond that, though, the vehicle needs to interpret the police officer's signals. If the police officer holds strictly to the rules, as shown in the photographs Figs. 7.4 and 7.5, an autonomous vehicle can learn the associated meanings. Experience has shown, however, that police officers directing traffic or parking lot attendants assigning parking spaces use very dynamic gestures to speed up traffic, such as waving their arms or hands or making rowing motions with their arms. See Figs. 7.6, 7.7 and 7.8. Humans usually understand these signals from context. Alongside the ability to recognize patterns of gestures and postures, autonomous vehicles would need to have contextual knowledge allowing them to identify and evaluate gestures correctly.

One exceptional case is the need to accommodate vehicles with special rights, such as police cars, fire trucks, or ambulances. These vehicles draw attention to themselves using auditory signals. Visual and auditory signals (such as sirens and flashing lights) obligate the rest of traffic to stop, cede right of way at an intersection, or move aside to create a rescue lane. In everyday road traffic, vehicles do not stop right away when they hear this type of auditory signal in the distance. Such a reaction would severely inconvenience traffic, especially near a hospital for instance. It is also impossible from a distance to precisely identify the direction the auditory signal is coming from. Likewise, the 


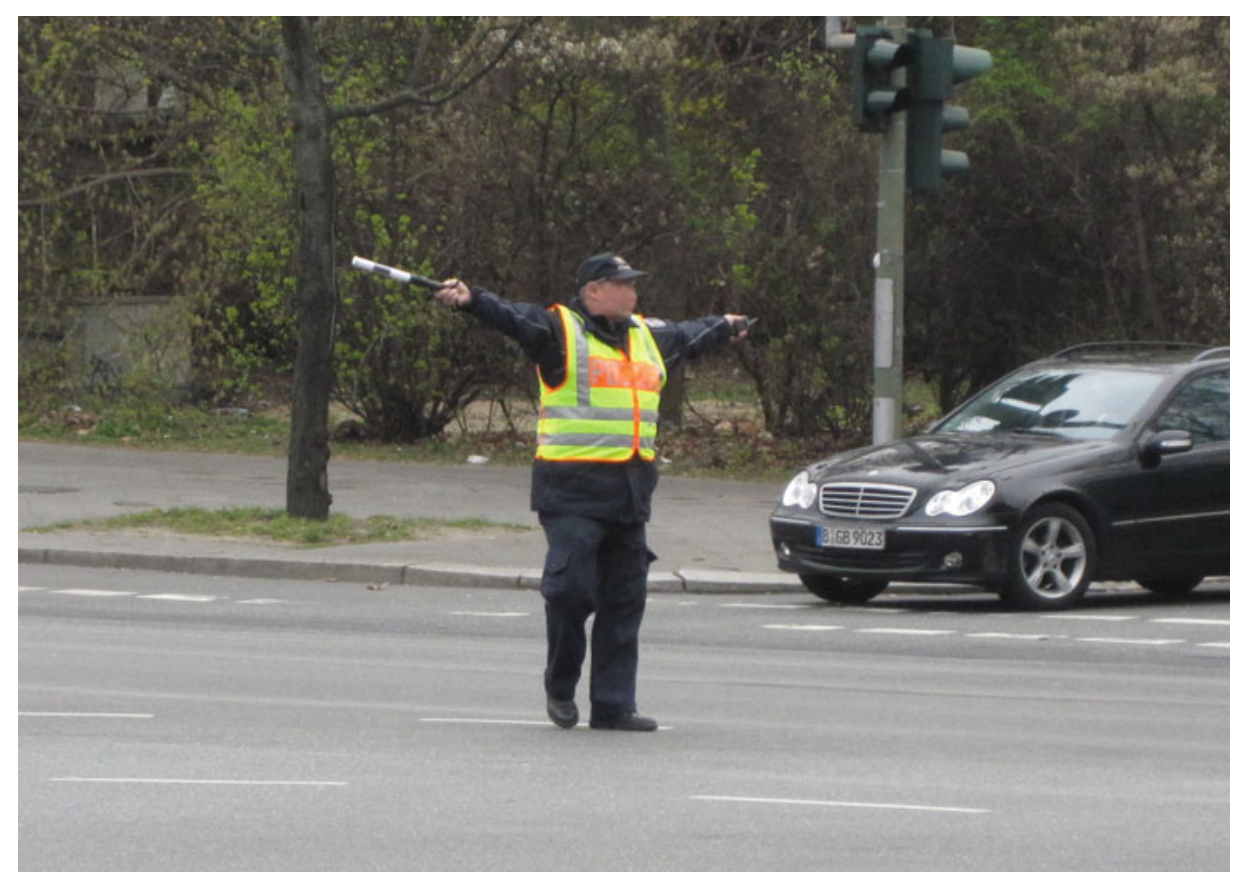

Fig. 7.4 Traffic police officer: traffic flowing. Image rights: http://commons.wikimedia.org/wiki/ User:Video2005? uselang=en

Fig. 7.5 Traffic police officer (North Korea): chest facing driver means "stop". Image rights: https://www.flickr.com/ people/kansai/

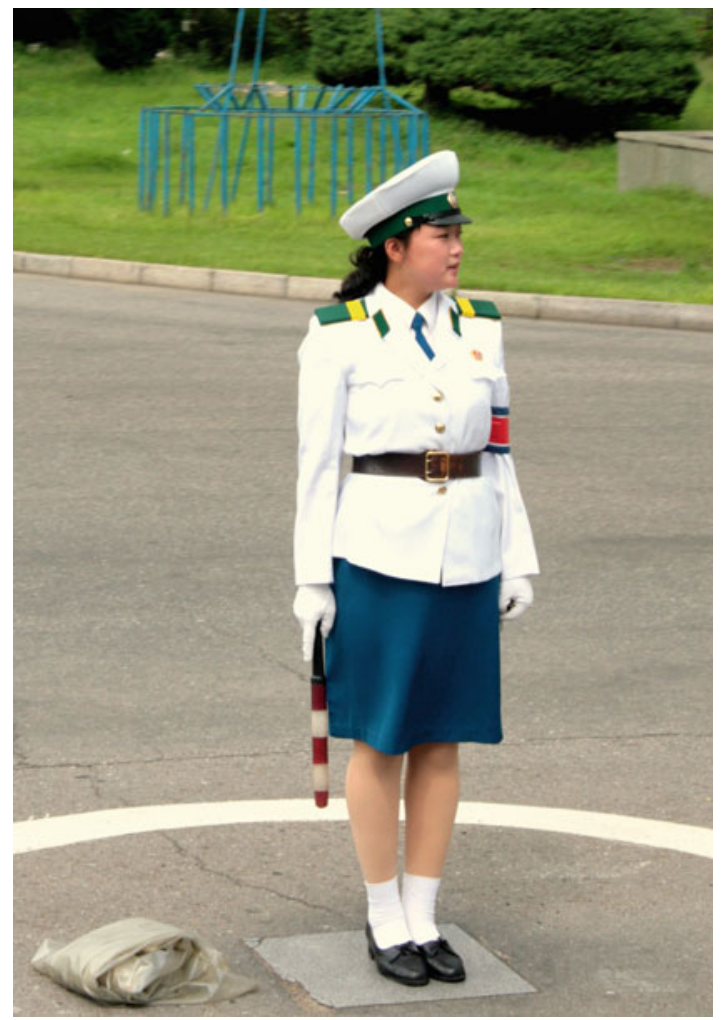




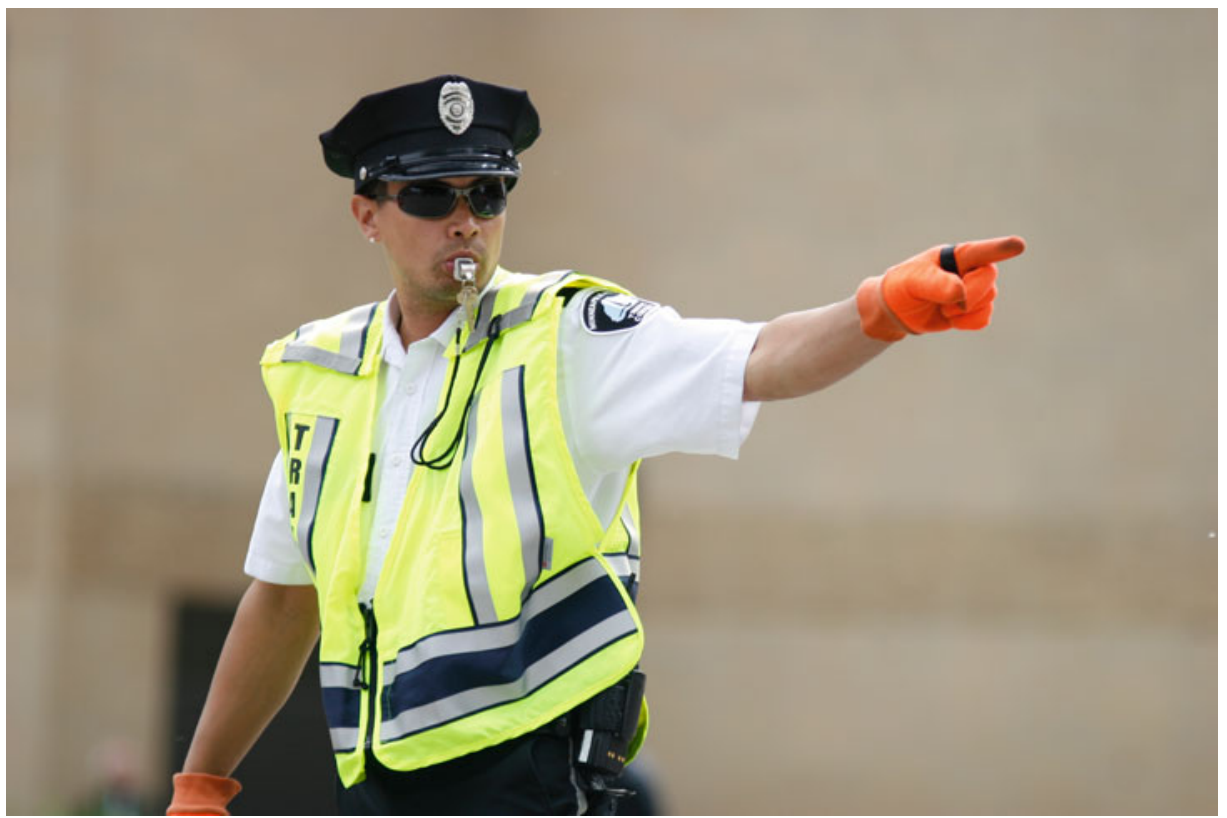

Fig. 7.6 Police officer in Minneapolis signals that traffic can start moving on one side of the street. Image rights: http://commons.wikimedia.org/wiki/User:Calebrw?uselang=en

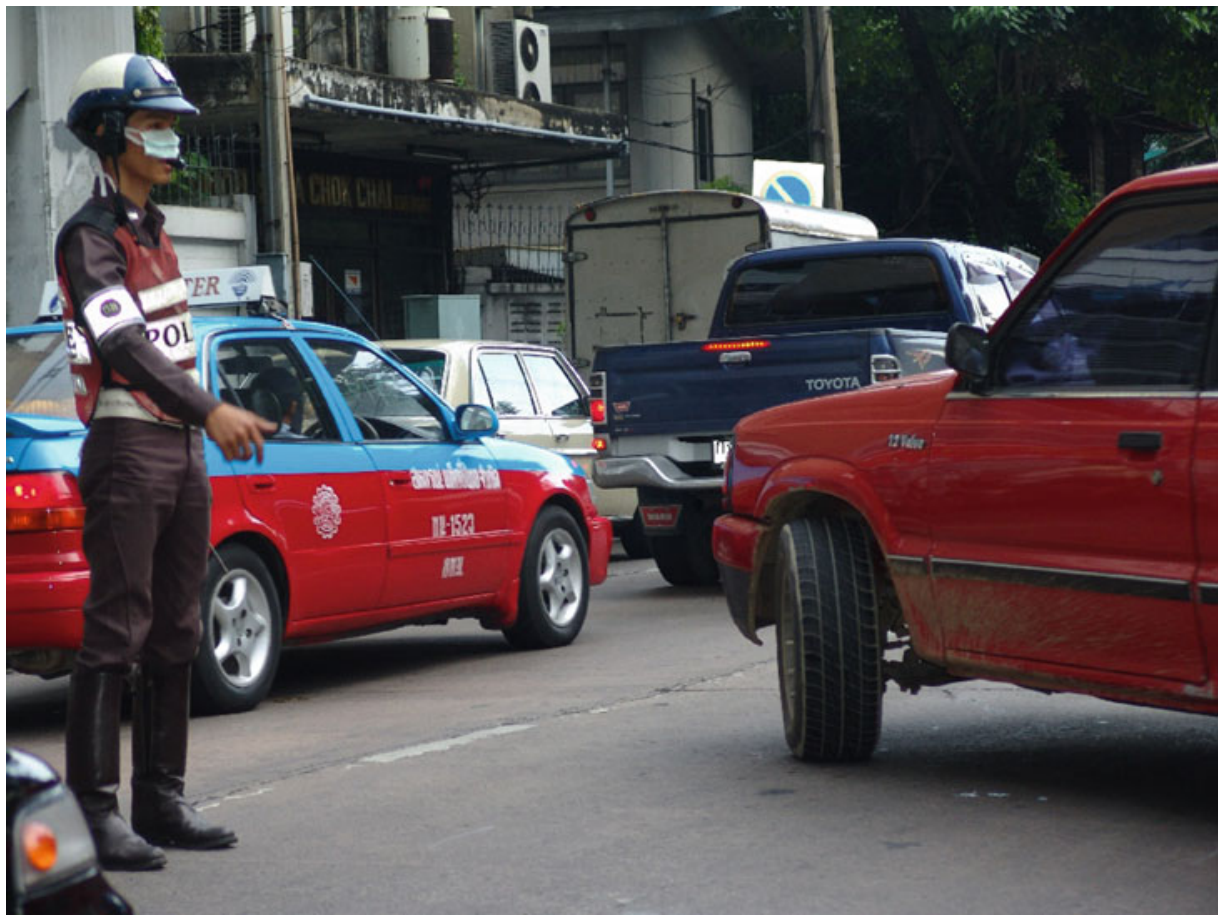

Fig. 7.7 Police officer in Bangkok gestures to a vehicle to turn. Image rights: http://de.wikipedia. org/wiki/User:Da?uselang=en 
Fig. 7.8 Police officer (Sweden) gestures to vehicle to move forward. Image rights:

Olle Nebendahl

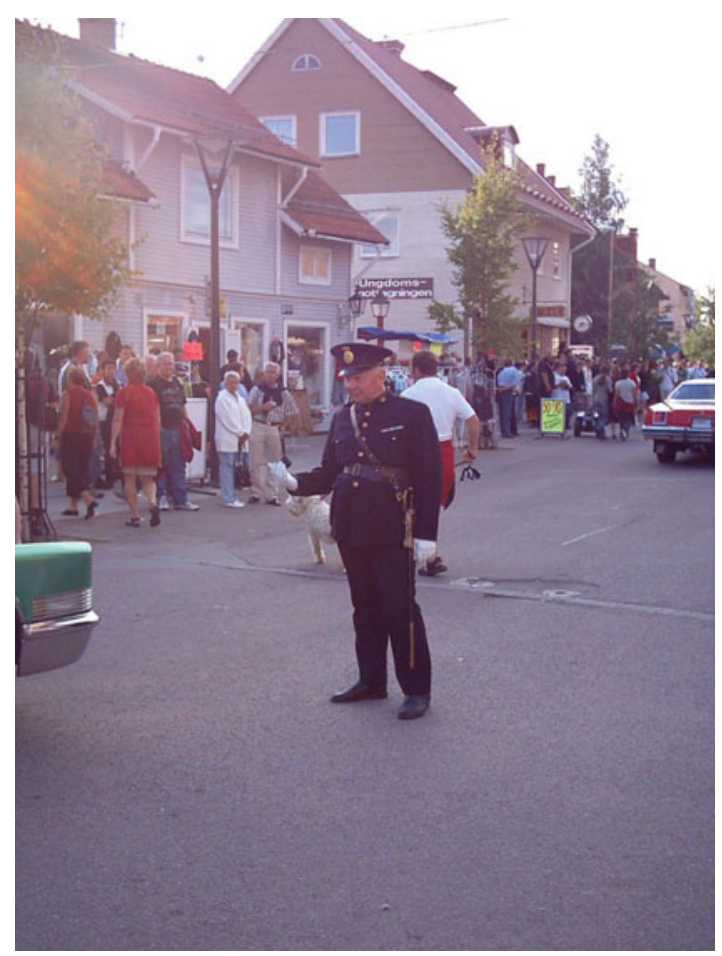

functionality to identify special vehicles from a distance and to determine the best way to rearrange the lanes would be difficult to design. For that reason, autonomous vehicles would need to stop upon all of these signals for safety reasons so as not to put traffic in danger. The following section will delve more deeply into the implications of the above on public acceptance.

\subsection{Is the Ability to Communicate a Requirement for the Other Road Users to Accept Autonomous Vehicles?}

In terms of public acceptance, we must once realize again the effect of informal communication in "negotiating situations." In keeping with the aforementioned Sect. 1 of the Highway Code, drivers communicate in negotiating situations in order to resolve situations that are rule-governed in principle but where blindly following the rules would disturb traffic considerably.

\section{Example 1}

If a vehicle is parking or idling in your lane, it is sometimes necessary to cross over to the opposite lane and, in extreme cases, even to cross a solid center line. In busy traffic, this 
requires coordinating with the oncoming vehicles. Apart from (unauthorized) crossing of the solid center line, the situation can be solved without a "negotiation" if oncoming traffic becomes sparse enough that the opposite lane can be used without vehicles communicating. The busier the traffic, the more likely a negotiation is necessary.

How does the driver of an oncoming vehicle signal willingness to cooperate? First of all, by slowing down in order to leave room for you to cross onto the opposite lane. Yet in most cases slowing down alone is not enough of a signal because there are many reasons why a vehicle might keep a larger-than-usual following distance. That is why approaching drivers would usually signal this kind of offer by both slowing down and flashing their headlights. If the autonomous vehicle did not recognize this signal, it would be seen as a "traffic obstacle," which would certainly not encourage public acceptance.

\section{Example 2}

As shown above, autonomous vehicles will generally behave more cautiously than human drivers as they have a limited grasp of the context and informal signals. Yet they can also perform driving maneuvers that are normally not performed by a human driver. For one thing, they react more quickly than human drivers. Since they tend not to have a "moment of shock," their reaction time is much faster. That is one reason why autonomous vehicles would cause fewer accidents. Furthermore, accident research has shown that many drivers do not make the most of their cars' braking capabilities and, even when swerving, tend not to reach the physical limits based on the circle of forces. Driver assistance systems such as automatic emergency brakes or emergency steer assist are intended to compensate for these deficiencies. On that basis, not only would an autonomous vehicle act more quickly in an emergency situation, but it would be able to take advantage of the physical limits of forward and lateral acceleration. How other road users will react is largely unknown at this point. The following section discusses a simple approach to solving this.

\subsection{What Mental Model Will Other Road Users Apply When Reacting to Autonomous Vehicle's Driving Errors?}

Closely related to the questions of interaction and negotiation discussed earlier is the issue of compensating for other road user's errors. Apart from avoiding immediate collisions with swerving trajectories, accommodating other driver's errors is normal in everyday road traffic. First let us clarify how "driving errors" is defined. From a human driver's perspective, "driving errors" might include purely rule-abiding behavior that is not ideally adapted to the situation.

For example, a human-driven car with right-of-way on a main road might let an autonomous vehicle from a side street turn first if the human-driven car wouldn't otherwise be able to turn onto the side street. In other words, if the human driver yields despite 
having right-of-way, the autonomous vehicle would need the ability to recognize that reliably.

Driving errors might also result from deficiencies in the autonomous vehicle's repertoire of strategies for resolving special situations or might be caused by reaching system boundaries.

\section{Example}

An autonomous vehicle operating in "traffic jam pilot" mode enters a safe state because the system's boundaries have been reached. In other words, the vehicle slows down to a stop. Although slowing down to a stop is common in a traffic jam, the other road users will be confused at the least if all other vehicles are moving and only the autonomous vehicle is stopped. If the maneuver occurs fairly abruptly for a reason that other drivers do not understand, this can pose a danger. However, we should keep in mind that road users do not drive without errors either in current road traffic and other drivers usually compensate for their errors. This raises the question of which characteristics humans will ascribe to autonomous vehicles. Will they be seen as less competent than human drivers or treated as flawless machines?

One goal of a deployment strategy must be to disseminate a positive yet realistic conception of autonomous vehicles among all road users. Then, and only then, will autonomous vehicles play an appropriate role in the traffic system. In general, the conditions for this are in place. For example, surveys of new technologies such as robotics have shown that Europeans have positive attitudes towards robots [12]. Likewise, driver assistance systems - as precursors of highly automated and ultimately autonomous vehicles - already enjoy a strong reputation as useful aids and are seeing increasing demand from consumers [13].

Apart from people's general attitudes towards technological systems, the capabilities and characteristics ascribed to new technological systems depend on the user's level of background knowledge. People who are less well-versed technically tend to apply naïve behavioral models that ascribe more capabilities to technological systems than they actually possess. Current demonstrations of autonomous vehicles in the media, largely conducted for marketing purposes, invite the impression that these vehicles are masters of all situations. This raises the expectations of autonomous vehicles so high that any driving errors would cause at least irritation, if not safety concerns. For that reason, in order to generate a realistic understanding of potential issues, it is essential to publicize autonomous vehicles' capabilities and limitations early on. 


\subsection{Cultural Differences}

A number of questions arise related to cultural differences: Are there universal rules for non-verbal behavior that can simply be adapted? How can cultural differences in communication and expectations be reflected in a robotic vehicle's communicative and decision-making behavior? If they can, how can they be adapted? What modes of behavior are there in different countries?

The best-known culturally comparative studies on non-verbal "utterances" based on facial expressions were produced by Ekman [14]. Across cultures, he found common facial expressions for the basic emotions of fear, disgust, happiness, sadness, surprise, anger, and contempt.

Most of these basic emotions do appear in road traffic, but their significance for communication among vehicles is rather restricted. For instance, the gestures for yes and no are crucial in negotiation situations. In central and northern Europe as well as the US, nodding means "yes" and shaking one's head means "no." However, in countries such as India, Pakistan, and Bulgaria, wobbling one's head sideways, a motion akin to the European and American "no" gesture, actually signifies agreement. Finally, there is an additional way to indicate yes and no non-verbally in countries such as Greece, Turkey, and southern Italy. "'Yes' is expressed by tilting the head forward, while 'no' is indicated by tilting it back" ([15], p. 134). Beckoning gestures are another source of misunderstandings during interactions among road users. A "paddling gesture" [15] with the palm facing down is used in Japan and the Mediterranean region for beckoning. In Germany and the UK, the same gesture is used to mean "go away." Despite the cultural differences among non-verbal signals performed with the hands, the gestures used to insult or rebuke other people in road traffic are largely universal, interestingly enough. Raising an index finger (Fig. 7.9), tapping on one's temple (Fig. 7.10) or making a sweeping motion beginning at the temple (Fig. 7.11) conveys one's contempt for the recipient. One exception is that forming an upright circle with one's thumb and index finger means (mostly) "o.k."in Germany, whereas in Italy it is understood as a gesture of insult (asshole) (Fig. 7.12).

In contrast to the pan-cultural basic emotions and "gestures of frustration," there are certainly cultural differences in informal communication among road users that are expressed in driving behavior and are currently in flux.

It is generally agreed that southern Europeans drive both more assertively and more defensively than central Europeans. In southern Europe, acceleration and the horn act as informal signals among vehicles. A driver merges into a lane of traffic by accelerating rapidly into a gap (potentially while honking the horn), expecting that the other road users will yield. The driver does not expect any other feedback, but simply assumes that the intention to merge will be recognized and accepted.

In central or northern Europe, the driver of the merging vehicle would tend to expect feedback in the form of eye contact at the least, if not a nod or a hand gesture. In Germany, 


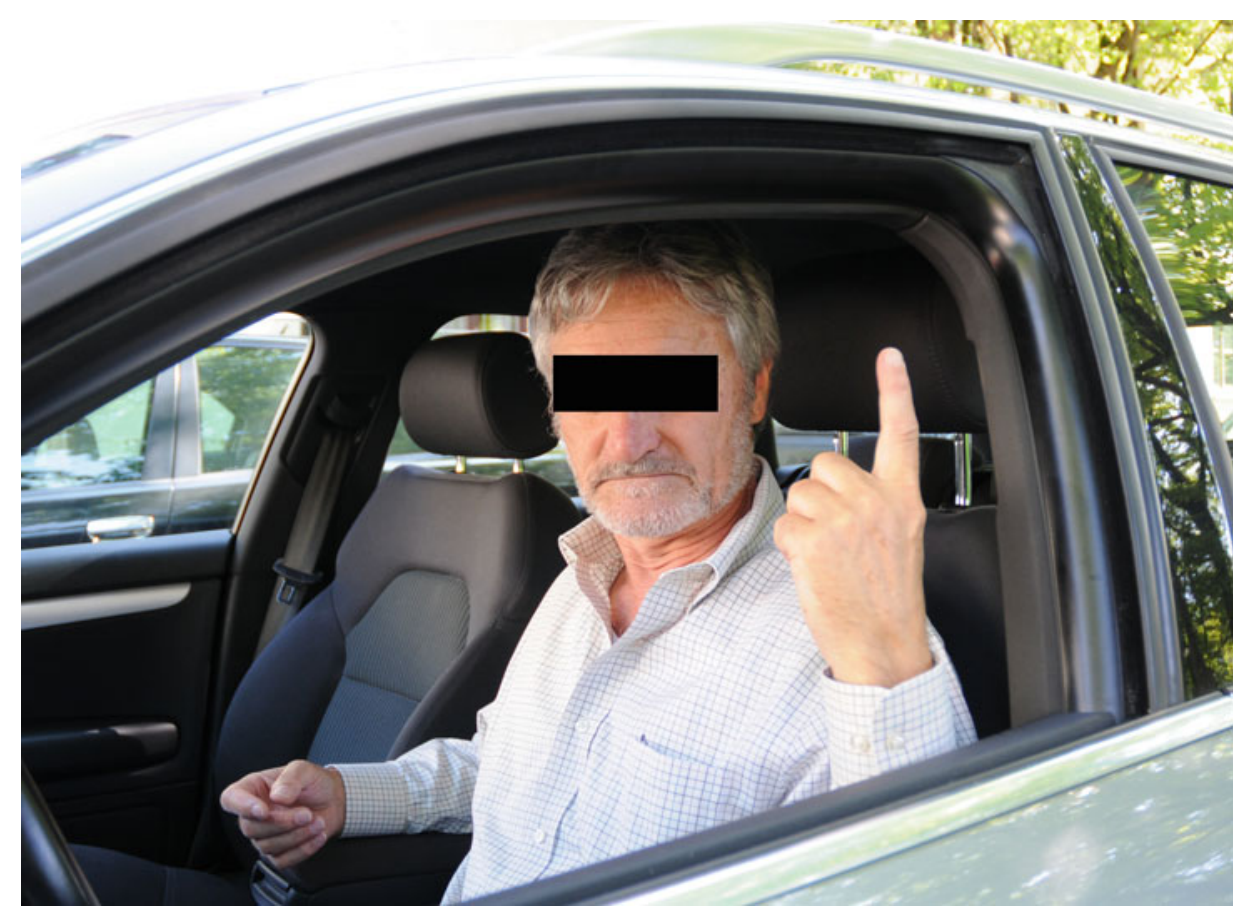

Fig. 7.9 Lifted finger. Image rights: copyright belongs to author

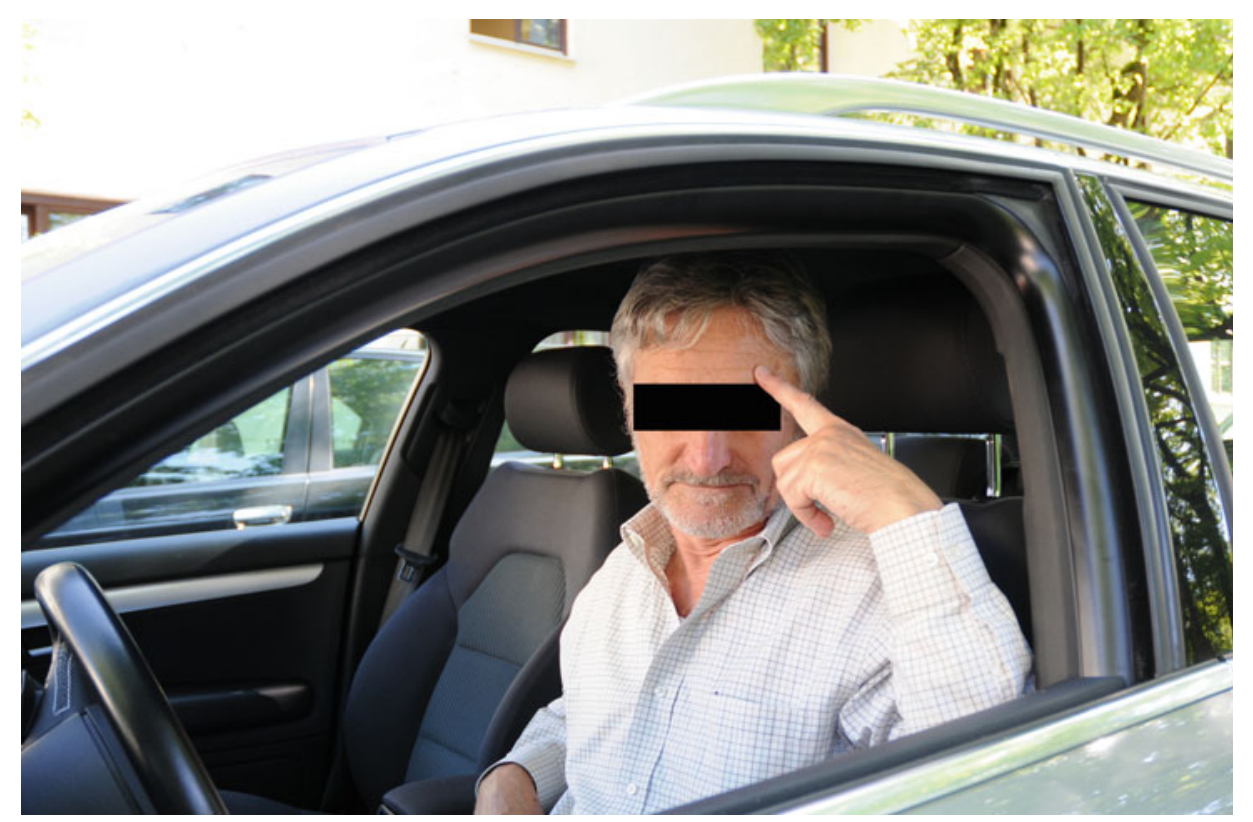

Fig. 7.10 Expressing frustration. Image rights: copyright belongs to author 


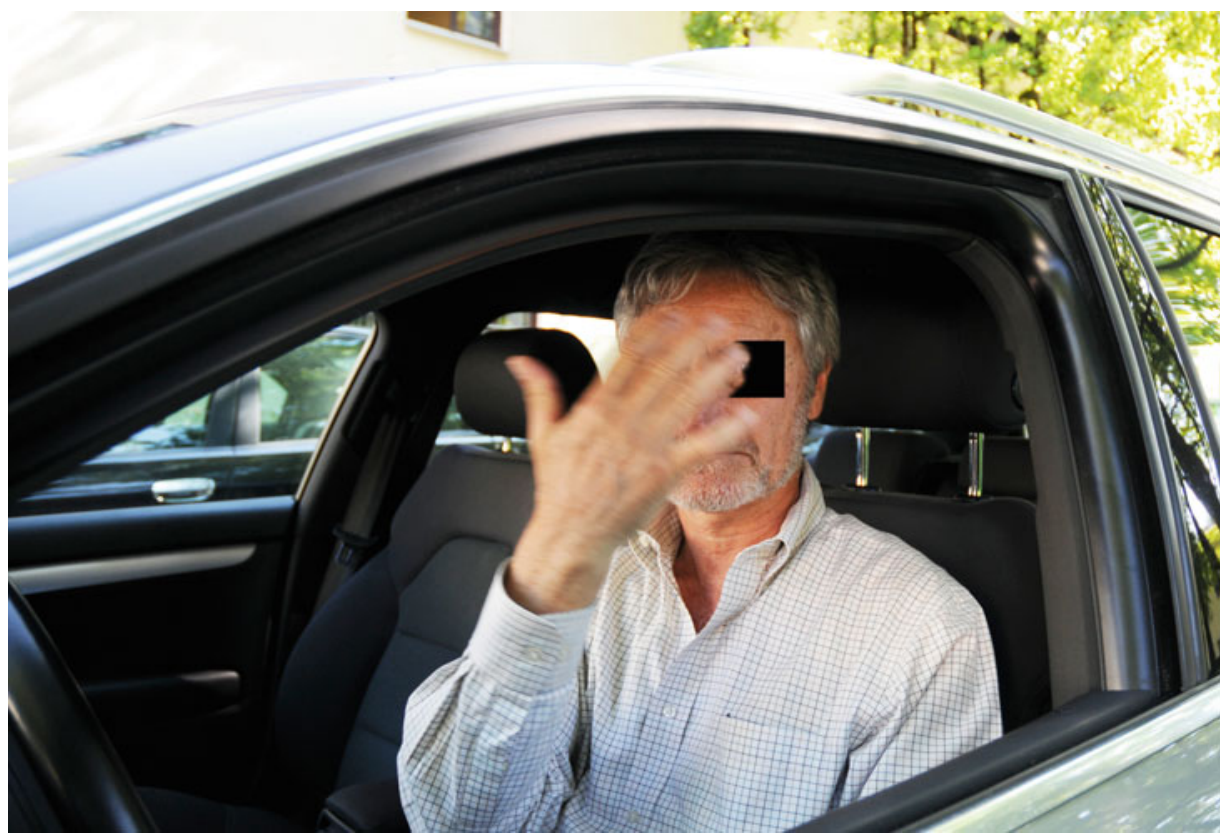

Fig. 7.11 A sweeping motion beginning at the temple to indicate bewilderment at the other person's behavior. Image rights: copyright belongs to author

at any rate, it is commonplace to insist on one's right-of-way so it would be unusual to attempt to merge without obtaining the other road user's "consent," as that would involve risking a collision.

Traffic in the United States is characterized by more steady, lane-based driving. Aside from the official signals, informal signals play less of a role there.

Traffic in China is - at least so far-marked by a low level of rule-abidance and by communication that is difficult for foreigners to interpret. Chinese drivers are masters of surprise, ignoring traffic rules and honking merely as a "friendly greeting" [16].

Communication between cars and pedestrians is especially critical.

Pedestrians make themselves understood by gesturing with their hands, stepping onto the crosswalk, or waiting there until a car stops. What are the implications of this for the behavior of autonomous vehicles? For one thing, autonomous vehicles will predict whether a pedestrian intends to cross the street based on the pedestrian's trajectory and acceleration. Apart from that, there are other modes of behavior that are more ambiguous, such as standing at a crosswalk without intending to cross (e.g. while having a conversation) or hesitating without making a clear signal of intending to cross. In all of these cases, the autonomous vehicle will need to stop for safety reasons. A consequence of this may be "unwarranted stopping" or the danger that children or teens catch on and begin to force vehicles to stop at crosswalks for fun. 


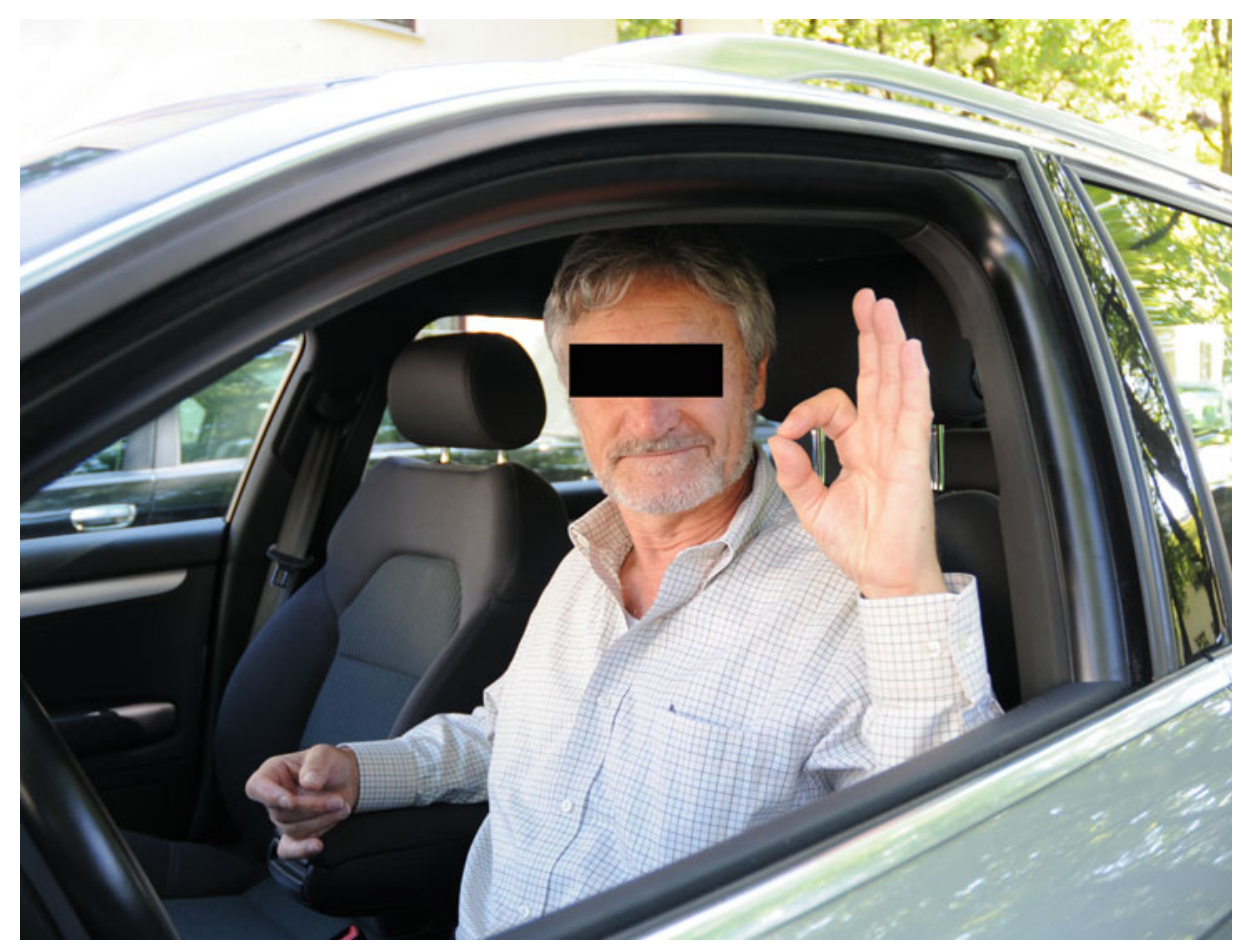

Fig. 7.12 No worries! Image rights: copyright belongs to author

Yet the regulations and the actual behavior alike vary widely from country to country. For instance, Italy did not have a rule requiring cars to stop at crosswalks until recently. According to reports from visitors to China, it is only advisable to cross at a crosswalk there as part of a crowd because traffic is highly unlikely to stop for a lone pedestrian.

While pedestrians who use hand gestures to communicate assume that their gestures are seen, they usually would not cross the street if an oncoming car did not slow down.

\subsection{Means of Compensation}

In the first stage, there is an easy antidote to the dilemma of robotic vehicles' inability to communicate informally in "negotiation situations."

Clearly and visibly marking autonomous vehicles as such would announce the vehicles' uniqueness to the other road users and make their deviant behavior more understandable. This would indicate to other road users that they cannot expect the usual behavior and would consequently increase acceptance in the sample situations described above. After all, vehicles for learner drivers are identified very visibly in order to inform other road users and excuse behavior that might be either excessively rule-abiding or overly hesitant. 
Especially in the introductory phase, several considerations support including identifying markings. If vehicles drive without a person sitting in the driver's seat, as envisioned by the "valet parking" scenario, this might irritate the other road users. "Is that car moving autonomously or is it just driving out of control?" With identifying markings, there is no need to ask. Marking autonomous vehicles can also have a marketing effect that will help give rise to rapid adoption. For example, when ABS was introduced, there were ABS stickers available that read "This vehicle has ABS brakes" to indicate that the braking distance was shorter. Technically that is not quite correct because ABS mainly makes it easier to steer while braking, but from a marketing viewpoint the sticker was a great success.

There are also some reasons not to give autonomous vehicles identifying markings. Because they must strictly adhere to the rules, due to their limited ability to negotiate among other things, they might also be targets for unwanted external interventions.

\section{A simple example:}

Pedestrians would not cross a street if a car was approaching due to uncertainty as to whether the car sees them or whether it will stop. In the case of an autonomous vehicle, pedestrians can be positive that the vehicle will stop no matter what (within physical limits). As a result, stopping autonomous cars could become a game for teenagers or cause adults to cross the street without paying attention to the flow of traffic. "It has to stop-it's programmed that way." Neither development would be good for the flow of traffic and this would not have a positive affect on the acceptance of autonomous vehicles.

Whether it has an outright negative effect is hard to predict. That is highly dependent on how exactly autonomous vehicles are introduced. If they are seen as a positive technological innovation and excused for having certain imperfections, a negative effect would be scarcely likely. On the other hand, if they are seen as an elite status symbol, envy will predominate and attempts to disrupt the system will be more common.

\subsection{New Forms of Communication for an Effective Exchange of Information from Both Psychological and Technological Perspectives}

Autonomous vehicles must generally be able to recognize and interpret other road users' gestures and trajectories. In order to interpret them, they will need situational knowledge allowing them to put signals in the correct context. The "official" signals such as turn signals, the horn, and headlight flashing would probably suffice in most situations in order to convey information to other vehicles or road users.

Kent Larsen's research group at MIT proposed an interesting approach for communication between autonomous vehicles and pedestrians [17]. A prototype with a variety of sensors but only remotely resembling an actual car was fitted with a number of devices. Swiveling, blinking LEDs designed to look like an eye turn towards a pedestrian, signaling "I've seen you." 
Additionally, directional speakers point towards pedestrians and tell them that it is okay to cross the street.

If any pedestrians are identified, color-coded LEDs in the wheels could change from green to yellow to red in order to acknowledge pedestrians or warn them (Fig. 7.13).

Another signal that autonomous vehicles can use to communicate with others is "explicit driving."

For example, if another vehicle would like to merge and the autonomous vehicle wants to cooperate, the autonomous vehicle should visibly slow down in order to accentuate the gap and assure the other driver that it is safe to merge.

Communication and informal rules are in constant flux. To illustrate this point with a verbal example, take the expression that something "sucks." Not too long ago, this word was understood as a direct sexual reference, provoking understandable consternation when young people began generalizing it. Similarly, the non-verbal signal "thumbs up" recently spread to countries where it was unfamiliar due to its iconic use in social media. From one angle, that means that autonomous vehicles will need to periodically learn new signals of non-verbal behavior, for instance by regularly updating their visual "vocabulary" along with associated meanings. Yet another effect can also be anticipated. The increased presence in road traffic of autonomous vehicles practicing a distinct, particularly rule-based set of behavior could also encourage other road users to adopt such behavior. This would make traffic more standardized, but not necessarily more efficient. Flexible cooperation between road users becomes more important when the traffic is busier. The distances narrow between vehicles, the complexity increases, and more informal signals are exchanged.

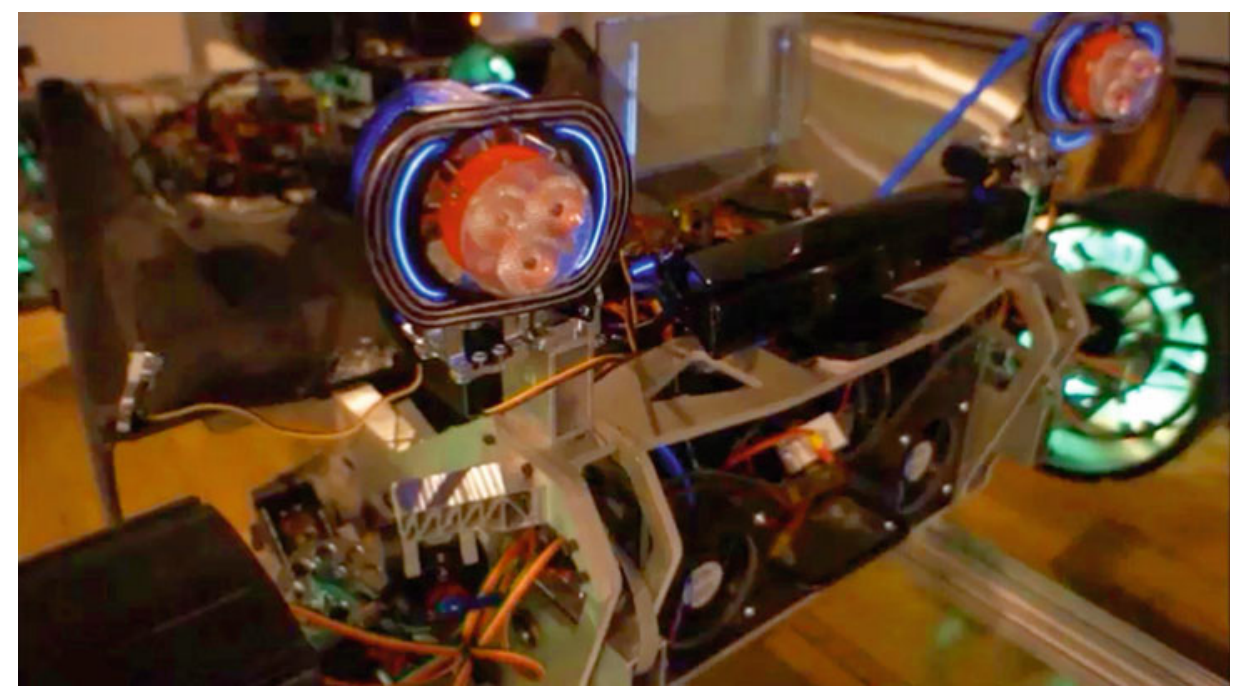

Fig. 7.13 AEVITA: Autonomous Electric Vehicle Interaction Testing Array. Image rights: Nicholas Pennycooke, Changing places group, MIT Media Lab (copyright belongs to author) 
Car-to-car communication could provide another technological solution. This would require all road users - not only autonomous vehicles — to be fitted with the corresponding technology. Car-to-car communication has undergone years of research and there have already been a number of demonstrations, some of them on large test sites such as simTD [20]. The proliferation of vehicle-to-vehicle (V2V) or vehicle-to-X (V2X, vehicle to infrastructure) technology could significantly facilitate solving the communication issues between autonomous vehicles and human drivers.

Frost \& Sullivan [21] project that by the year 2030, $40 \%$ of vehicles will be equipped with $\mathrm{V} 2 \mathrm{~V}$ or $\mathrm{V} 2 \mathrm{X}$ technology due to the major advantages these technologies offer users, including reducing traffic jams and enhancing safety. The rapid spread of this technology, initially with a different focus in mind, could thus mitigate communication problems between autonomous vehicles and those driven by humans.

Yet even then there will still be some situations that autonomous vehicles cannot solve, either because the other road users do not have the necessary communication devices or because the situation cannot be resolved through communication alone. If the autonomous vehicle operates without a driver to take over driving on short notice (although not immediately), a remote driver or guidance system would have to step in.

\subsection{Conclusions}

Because vehicle-to-vehicle communication options are currently very limited and unreliable, a wide variety of glances, actions, and action sequences are used to communicate with unprotected road users. Depending on the circumstances and the state of mind of the people involved, interpersonal negotiations can have varying levels of intensity. The rules applied in these cases are highly culturally specific. In particular, the expectations placed on the other person vary widely from culture to culture, so it would not be possible to compile universal rules for robotic vehicles. Another problem arises merely due to the fact that the vehicle's driver, the robot, is unrecognizable. This is evident for driverless cars, at any rate. If a person is sitting in the driver's seat of the autonomous vehicle, any communication would likely be misdirected at the presumed human driver. Mixed traffic including cyclists and other vehicle types would be difficult to implement without the ability to recognize other road users' glances and gestures. This is especially true in low-speed settings. As the speed increases, this form of communication plays a less significant role for a variety of reasons:

- It is not $100 \%$ explicit.

- It requires feedback from the other person, which would take too long.

- At high speed there is a limited ability to assess the other road user's reaction. There is not enough time to evaluate another driver's gaze, for instance.

What requirements does this create for ensuring functionality based on communication with other road users? 
As such, autonomous vehicles must initially behave as if there were no informal communication, in other words, abiding completely by the rules. In the event of an unresolvable situation, either a human driver must take over, or in the absence of one, a centralized authority would need to intervene. Traffic management would be organized similarly to air traffic, in which air traffic control coordinates all aircrafts' movements both in the sky and on the tarmac. Instead of a pilot executing air traffic control's instructions, the robotic vehicle would receive instructions about how to maneuver and independently ensure the vehicle's stability as it carried them out. It remains unclear how an autonomous vehicle would recognize that the situation cannot be resolved or how the traffic control authority would be informed of the relevant details.

Open Access This chapter is distributed under the terms of the Creative Commons Attribution 4.0 International License (http://creativecommons.org/licenses/by/4.0/), which permits use, duplication, adaptation, distribution and reproduction in any medium or format, as long as you give appropriate credit to the original author(s) and the source, a link is provided to the Creative Commons license and any changes made are indicated.

The images or other third party material in this chapter are included in the work's Creative Commons license, unless indicated otherwise in the credit line; if such material is not included in the work's Creative Commons license and the respective action is not permitted by statutory regulation, users will need to obtain permission from the license holder to duplicate, adapt or reproduce the material.

\section{References}

1. archive.darpa.mil/grandchallenge/ last accessed on 25.7.2014

2. Ziegler, J., Bender, P., Lategahn, H., Schreiber, M., Strauß, T., Stiller, C. (2014). Kartengestütztes automatisiertes Fahren auf der Berta-Benz-Route von Mannheim nach Pforzheim. FAS 2014, Uni-DAS, pp. 97-94

3. Saust, F., Wille, J.M., Lichte, B., Maurer, M.: (2011). Autonomous Vehicle Guidance on Braunschweig's inner ring road within the Stadtpilot Project. In: Intelligent Vehicles Symposium (IV), Baden-Baden. pp. 169-174

4. Merten, K. (1977). Kommunikationsprozesse im Straßenverkehr. In: Bundesanstalt für Straßenwesen (ed.) Symposion 77. Köln: Bundesanstalt für Straßenwesen

5. Darwin, C.: Der Ausdruck der Gemüthsbewegungen beim Menschen und den Thieren. Halle 1874

6. Pfungst, O.: Das Pferd des Herrn von Osten (Der kluge Hans). Leipzig 1907

7. Schmidt, S., Färber, B. (2009). Pedestrians at the kerb - Recognising the action intentions of humans. Transportation Research Part F: Traffic Psychology and Behavior, 12, pp. 300-310.

8. Risser, Ralf.: Kommunikation und Kultur des Straßenverkehrs. Wien 1988

9. Heidegger, Martin (1927). Sein und Zeit. Tübingen. 11. Auflage 1967

10. Frerichs, Klaus (1995). Der Winker und das Winken: Ein zeichenphänomenologischer Passus in Martin Heideggers Sein und Zeit. Zeitschrift für Semiotik, Band 17, Heft 1-2. pp. 133-142

11. Savigny, E.v. (1995). Autofahrerzeichen: Funktion, Systeme, Autonomie. Zeitschrift für Semiotik, Band 17, Heft 1-2. pp. 105-128

12. Special Eurobarometer 382, Public Attitudes Towards Robots, Report Sept. 2012

13. Continental Mobilitätsstudie http://www.pressebox.de/pressemitteilung/continental-reifen-deutschland-gmbh-hannover/Deutsche-Autofahrer-wollen-Automatisiertes-Fahren-fuer-die-Autobahn/ boxid/647170 last accessed on 26.8.2014 
14. Ekman, P. Universals and Cultural Differences in Facial Expressions of Emotion. In: J. Cole (Hrsg.): Nebraska Symposium on Motivation 1971. Bd. 19, University of Nebraska Press, Lincoln 1975.

15. Broszinsky-Schwabe, Edith (2011). Interkulturelle Kommunikation: Missverständnisse Verständigung. VS Verlag, Wiesbaden.

16. http://www.auto-motor-und-sport.de/news/autofahren-in-china-ein-mann-sieht-rot-5957313.html last accessed on 7.8.2014

17. http://www.technologyreview.com/view/427743/how-do-you-know-an-autonomous-vehicle-hasseen-you/ last accessed on 8.9.2014

18. ICTTP'08 - Proceedings of the International Congress of Traffic and Transport Psychology, Washington 2008

19. Hölzel, Andrea (2008) Unterscheidung von formeller und informeller Kommunikation im Straßenverkehr http://othes.univie.ac.at/2541/1/2008-11-12_9547261.pdf zuletzt besucht 29.07. 2014

20. simTD delibrable D5.5 (2013) http://www.simtd.de/index.dhtml/object.media/deDE/8154/CS/-/ backup_publications/Projektergebnisse/simTD-TP5-Abschlussbericht_Teil_A_Manteldokument_ V10.pdf

21. Strategic Analysis of the European Market for V2V and V2I Communication Systems (2014) cited from http://www.frost.com/prod/servlet/press-release.pag?docid=290129681 